\title{
Téoros
}

Revue de recherche en tourisme

\section{Les fêtes et les événements font-ils partie des pratiques touristiques des Québécois?}

\section{Pierre Bellerose, Andrée Lamontagne, Marc Laplante et Marie-Claude Cardin}

Volume 5, numéro 2, juillet 1986

Tourisme, fêtes et événements

URI : https://id.erudit.org/iderudit/1080579ar

DOI : https://doi.org/10.7202/1080579ar

Aller au sommaire du numéro

Éditeur(s)

Université du Québec à Montréal

ISSN

0712-8657 (imprimé)

1923-2705 (numérique)

Découvrir la revue

Citer cet article

Bellerose, P., Lamontagne, A., Laplante, M. \& Cardin, M.-C. (1986). Les fêtes et les événements font-ils partie des pratiques touristiques des Québécois?

Téoros, 5(2), 36-37. https://doi.org/10.7202/1080579ar 


\section{Les fêtes et les événements font-ils partie des pratiques touristiques des Québécois?}

\section{Les fêtes ou les événements comme motivation de voyages de vacances}

\section{par Pierre Bellerose et Andrée Lamontagne}

Les fêtes et les événements peuvent-ils jouer le rôle d'attractions touristiques? Sont-ils capables de justifier des déplacements de vacances?

Sans données à ce propos, nous ne pouvons. pas répondre à de telles questions. Aussi, en prévision du présent numéro de Téoros, nous avons procedé à un mini-sondage au kiosque de l'UQAM lors du dernier Salon Vacances Québec. Cent quatorze personnes ont répondu à un questionnaire distribué de façon non aléatoire't?. Les résultats resteront donc indicatifs et devront être confirmés par un sondage scientifique.

\section{Les fêtes ou les événements ne sont pas des attractions}

Comme en font foi les tableaux 1 et 2 , les fêtes ou les événements n'occupent pas une grande place dans les projets de vacances des Québécois ni dans le programme de leurs activités de voyage.

$8.8 \%$ des personnes interrogees ont choisi leur destination de vacances à cause d'une fểte ou d'un événement. Comme raison principale du choix d'une destination, on trouve d'abord la visite d'une région $(31.6 \%)$, la visite de parents ou d'amis $(25.4 \%)$ et le climat (20\%). Au cours de leur dernier voyage $18.4 \%$ des répondants ont, de fait, participé à une féte ou à un évétnement touristique.

Le public consulté a des habitudes de vacances assez conformes aux données connues sur cette clientèle scolarisée: $50 \%$ environ ont passé leurs vacances au Québec, $15 \%$ dans les autres provinces canadiennes, $11 \%$ aux USA et $24 \%$ hors de l'Amérique du Nord. Les fêtes et les evénements font partie des programmations touristiques en Europe, aux USA, au Canada comme au Québec. Si ces activi. tés êtaient considerées comme des attractions, elles auraient pu être fréquentées à toutes les destinations choisies.
Des habitudes à prendre ou une promotion à reprendre

Faut-il croire que les fêtes et les événements - pourtant souvent conçues pour les visiteurs - ne servent que de compléments au voyage de vacances? Une sorte de prime pour rehausser davantage les plaisirs des visites régionales, des rencontres parentales ou amicales?

Faut-il plutôt interroger l'information. la promotion ou la publicité touristiques? Si celles-ci intègrent bien les fêtes et les événements dans les invitations au voyage, il faudra en déduire que de telles expériences ne font pas encore partie des plaisirs attendus $\mathrm{d}^{+}$un voyage de vacances.

Mais comment les rapports de promotion et de publicité touristiques du Québec servent-ils les fêtes et les événements? Cette question fut a l'origine d'une autre petite etude à l'occasion du dernier Salon de Vacances-Québec.
Les fêtes et les événements - ponctuels ou cycliques - font toujours partie des menus de voyages de vacances: avec les activités spatiales, artistiques ou sociales de loisir. avec les monuments, les lieux historiques. I'architecture, les paysages ou les ressources naturelles, ils donnent aux touristes les motivations associes à une destination.

Québec, terre d'hospitalité dans une nature encore bien conservée, Québec avec un riche passế original qui n"a pas encore disparı derrière les façades du 20 e siècle.
Tableau 1

Raison principale du choix d'une destination

Visite d'une région touristique

Visite de parents-amis

Climat

Participation à une fête

ou un événement

20.2

Autres

Total

\section{Tableau 2}

Participation à une féte ou

à un événement au cours

du dernier voyage

\begin{tabular}{lc}
\hline Oui & 18.4 \\
Non & 81.6 \\
Total & 100.00
\end{tabular}

\section{Note}

(1) 639 avaient entre 18 at 34 ans. $60 \%$ etaient des femmes; $72 \%$ venaient du Montreal metropolitain. Ce public tah très scolarise: $76 \%$ avec etudes col. légiales el plus $141 \%$ avec scolarité universitairel.

\section{Les fêtes et les événements dans la promotion touristique au Québec}

\section{par Marc Laplante, Marie-Claude Cardin et Andrée Lamontagne}

Québec de la joie de vivre, comme on se plait à le dire, devrait offrir à ses visiteurs toutes les chances possible de s'associer la fëte. Avec plus de 25 événements majeurs par année - dont plusieurs mainte. nant sont annuels, avec pres de 200 fétes populaires, festivals et carnavals, avec une trentaine de musees affichant des expositions spéciales en haute saison, pour ne rien dire des programmes d'été de théátre, de concerts et de spectacles, on peut s'attendre à retrouver dans la promotion touristique de nombreuses incitations à participer à cette effervescence culturelle. 
Les fêtes et événements au Salon Vacances Québec 86

Pour vérifier l'importance donnée aux fêtes et aux événements dans la promotion tou= ristique, nous avons eu l'idée de sonder le dernier Salon Vacances Québec (17-20 avril 1986). Une petite équipe d'observateurs a parcouru l'aire d'exposition du Palais des congrès de Montreal pour simplement noter les régions selon qu'elles utilisaient-ou non- les fétes et les ếvênéments dans leur présentation.

Sur 185 exposants différents, 3 étaient exclusivement concernés par des fêtes ou des événements: le Festival de la crevette de Matane (F-6), le Festival international de la bicyclette de Hull (J-4) et le Festival mondial de folklore de Drummondville (L-14).

Par région, nous avons tênté d'estimer la plus occupée visuellement par le thème Fëtes et Evénements dans l'ensemble des sujets affichés dans un kiosque (y compris les dépliants et brochures ou les posters disponibles pour les visiteurs).

a) Certaines régions n'ont rien présenté concernant les fêttes et les événements se passant dans leur territoire: Charlevoix, Laurentides.

b) D'autres régions $n^{*}$ ont pas utilisé visuellement le thème des fêtes et des événements mais avaient des dépliants à ce propos: Portneuf (pour le Festival du lin à St-Léonard), Coeur du Québec (a cause du Festival de Drummondville), Montérégie (pour le Festival des mont" golfières), Lanaudière (pour le Festival de la musique), Gaspésie (pour Matane et aussi le Festival nautique de la Baie des Chaleurs), l'Abitibi-Témiscamingue et Duplessis.

c) Quelqués régions ont accordé de $10 \%$ à $25 \%$ de leur espace visuel à notre thème: le Bas St-Laurent (présentation peu stimulante: $15 \%$ de l'espace), I'Estrie (avec 3 localités et leur fêtesévenements: Thetford Mines, Granby, Asbestos et la ville de Sherbrooke en particulier - un dépliant spécial bien en vue), I'Outaouais (avec la "Bicyclette". mais aussi avec le Festival de voile à Aylmer et quatre événements pour la sous-région de Petite-Nation).

d) Montréal et Québec qui ont axé l'essentiel de leur promotion du Salon sur les fêtes et les événements.

e) Une seule région a misé fortement sur les fétes et les événements, celle du Saguenay-Lac-St-Jean-Chibougameau: dépliant exceptionnel présentant une quarantaine de manifestations sur les 12 mois de l'année, atmosphère de fête au kiosque, $50 \%$ à $60 \%$ de l'espace visuel dévolu à ces thèmes, etc...
Ce bilan nous a surpris en première analyse mais il $\pi$ 'est pas si étonnant. Les grandes villes sont en fait le lieu tout désigné pour tenir des fêtes ou des événements majeurs pour lesquels on peut investir en publicité. Les itinéraires de vacances croisent presque toujours Montréal et/ou Québec, pour les Québécois comme pour les étrangers: ces manifestations exigent souvent des ins. tallations qu'on ne trouve que dans les grandes villes, etc...

Pourtant, compte-tenu des fonds publics investis pour l'ensemble de ces fêtes et événements, considérant aussi la promotion qui les entoure hors du réseau de communications touristiques, nous devons constater que les responsables touristiques en région n'ont pas encore réellement intégré les manifestations culturelles - à l'exception du Saguenay-Lac-St-Jean - dans leur présentation.

\section{Les fêtes et les événements dans la publicité accompagnant le Salon}

Le Cahier Vacances-voyages de La Presse du 12 avril 1986 était conçu comme un support d'information et de promotion du Salon. Sur les 18 pages de textes et d'annonces publicitaires ( 2 pages n'appartenaient pas au sujet), les fétes et les événements n'occupaient qu'un espace ridicule:

a) Dans les textes, quelques lignes sur Bromont et la Coupe du Monde de Ski Alpin 86 et autant sur les festivités de l'Abitibi-Témiscamingue (tour cycliste, centenaire de Ville-Marie, Festival de cinéma) à l'occasion de la présentation de la nouvelle brochure touristique régionale.

b) Regardant, comme tout lecteur de ce Cahier, les annonces publicitaires, nous avons constaté d'abord que 60 des 85 annonces proposaient des voyages hors du Québec sans jamais souligner des fêtes et des événements ailleurs dans le monde (en France, en Grèce, en Italie, etc... l'été est l'occasion de centaines de manifestation culturelles souvent de caractère exceptionnel). Une seule exception dans ces 60 annonces: trois proposaient, parmi d'autres forfaits, un séjour à Vancouver pour Expo 86 (un mois avant son ouverture) dont une mentionnait aussi un arrêt possible pour le Stampede de Calgary. Enfin, sur les 25 annonces proposant des voyagesvacances au Québec, aucune ne faisait référence aux fêtes et aux événements québécois de l'été.

Pour faire une dernière vérification avant de conclure, un examen attentif du Cahier Vacances-voyages de La Presse du 7 juin 1986 confirmait les conclusions tirées de celui du 12 avril précédent: une demi-page sur Ottawa-Hull, assez remarquable, entiè-

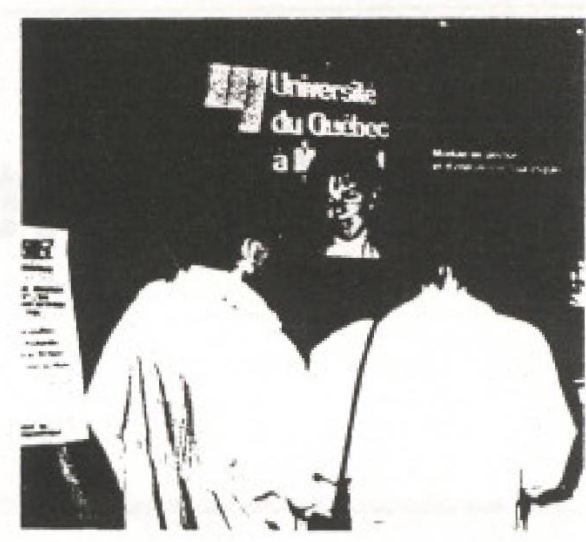

Kiosque de I'UAAM au Salon Vacances. Québec: un mini-sondage sur la place des fêtes et des évenements dans les projets de vacances des Québécois.

rement consacrée aux manifestations culturelles et sportives de l'été. Un grand centre urbain de plus mise sur les fêtes et les événements pour son tourisme!

Une analyse aussi sommaire n'autorise pas des généralisations, mais quelques constats s'imposent:

- L'information et la promotion touristique semblent se faire parallèlement à celles des manifestations culturelles; les parallèles, par définition, ne se rencontrent pas mais dans les grands milieux urbains, on note maintenant des convergences;

- On soupçonne, sans pouvoir l'affirmer, que certaines fétes ou certains événements sont beaucoup plus pour touristes que d'autres, une analyse proprement touristique de chaque manifestation devient de plus en plus nécessaire.

- Quand les fêtes et les événements prendront leur vraie place dans le développement touristique, il faudra investir beaucoup d'efforts pour la programmation à l'échelle du Québec. Déjà, semble-t-il, les seuls événements internationaux de mai et juin à Montréal cette année se sont nuis les uns les autres (chronique de Francine Grimaldi, La Presse, dimanche le 8 juin 1986). Au Saguenay-Lac-St-Jean, le dépliant sur les fêtes et les événements montre qu'il $y$ a eu programmation sur une base annuelle pour cette région, mais si les 17 autres font de mềme, la Fête comme attrait touristique au Québec sera-t-elle encore possible?

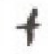

